\title{
Graded Neuronal Modulations Related to Visual Spatial Attention
}

\author{
다. Patrick Mayo ${ }^{1}$ and $\oplus^{\top} J o h n$ H. R. Maunsell ${ }^{2}$ \\ ${ }^{1}$ Department of Neurobiology, Harvard Medical School, Boston, Massachusetts 02115, and ${ }^{2}$ Department of Neurobiology, University of Chicago, Chicago, \\ Illinois 60637
}

Studies of visual attention in monkeys typically measure neuronal activity when the stimulus event to be detected occurs at a cued location versus when it occurs at an uncued location. But this approach does not address how neuronal activity changes relative to conditions where attention is unconstrained by cueing. Human psychophysical studies have used neutral cueing conditions and found that neutrally cued behavioral performance is generally intermediate to that of cued and uncued conditions (Posner et al., 1978; Mangun and Hillyard, 1990; Montagna et al., 2009). To determine whether the neuronal correlates of visual attention during neutral cueing are similarly intermediate, we trained macaque monkeys to detect changes in stimulus orientation that were more likely to occur at one location (cued) than another (uncued), or were equally likely to occur at either stimulus location (neutral). Consistent with human studies, performance was best when the location was cued, intermediate when both locations were neutrally cued, and worst when the location was uncued. Neuronal modulations in visual area V4 were also graded as a function of cue validity and behavioral performance. By recording from both hemispheres simultaneously, we investigated the possibility of switching attention between stimulus locations during neutral cueing. The results failed to support a unitary "spotlight" of attention. Overall, our findings indicate that attention-related changes in V4 are graded to accommodate task demands.

Key words: macaque; neuronal correlations; population coding; spatial attention; vision; visual area V4

\section{Significance Statement}

Studies of the neuronal correlates of attention in monkeys typically use visual cues to manipulate where attention is focused ("cued" vs "uncued"). Human psychophysical studies often also include neutrally cued trials to study how attention naturally varies between points of interest. But the neuronal correlates of this neutral condition are unclear. We measured behavioral performance and neuronal activity in cued, uncued, and neutrally cued blocks of trials. Behavioral performance and neuronal responses during neutral cueing were intermediate to those of the cued and uncued conditions. We found no signatures of a single mechanism of attention that switches between stimulus locations. Thus, attention-related changes in neuronal activity are largely hemisphere-specific and graded according to task demands.

\section{Introduction}

Many studies of spatial attention cue the subject to attend to the location of a to-be-judged task event, such as a change in stimulus orientation (Posner, 1980). The behavioral effects of attention are measured by comparing performance on "cued" trials, in which the event occurs at the cued location, against infrequent "un-

Received Jan. 18, 2016; revised April 2, 2016; accepted April 6, 2016.

Author contributions: J.P.M. and J.H.R.M. designed research; J.P.M. performed research; J.P.M. and J.H.R.M. analyzed data; J.P.M. and J.H.R.M. wrote the paper.

This work was supported by National Institutes of Health Grant R01EY005911 and National Institutes of Health Fellowship F32EY02259 to J.P.M.. We thank Thomas Luo and Bram-Ernst Verhoef for comments on the manuscript; and Steven Sleboda for technical assistance.

The authors declare no competing financial interests.

Correspondence should be addressed to Dr. J. Patrick Mayo, Duke University, 327B Bryan Research Bldg, Box 3209, 311 Research Drive, Durham NC 27705. E-mail: mayo@neuro.duke.edu.

DOI:10.1523/JNEUROSCI.0192-16.2016

Copyright $\odot 2016$ the authors $\quad 0270-6474 / 16 / 365353-09 \$ 15.00 / 0$ cued" trials, in which the event occurs at the unexpected location. Cueing is typically associated with superior performance, measured as success in detecting changes, lower thresholds for perception, or faster reaction times (Carrasco, 2011). Cueing therefore experimentally controls the focus of visual attention.

Studies of the neuronal mechanisms of spatial attention have relied on cued stimulus locations for decades (Moran and Desimone, 1985; Spitzer et al., 1988; Connor et al., 1996; McAdams and Maunsell, 1999). But human psychophysicists also frequently include an ambiguously cued, or neutral, condition where the task event is equally likely to occur at either location. The neutral condition captures the uncertainty that frequently occurs in natural environments, making it a useful tool for testing hypotheses about attention-related neuronal modulation.

Human performance in the neutral condition is usually better than the uncued condition and worse than the cued condition 
(Posner et al., 1978; Posner, 1980; but also see Posner et al., 1980; Proverbio and Mangun, 1994; Mangun and Buck, 1998; Montagna et al., 2009). Performance is best when the focus of attention is at the location of the stimulus change, and worst when the focus of attention is directed away from the location of the stimulus change. Human fMRI experiments have shown that performance in the neutral condition does not arise from an expanded attentional spotlight that covers the relevant stimuli and the visual field between them (Somers et al., 1999). Enhanced neural responses are instead concentrated in the cortical regions that represent the stimuli. Correspondingly, single-unit experiments in monkeys have shown that neuronal responses can be associated with spatially separated representations (Niebergall et al., 2011).

Behavioral performance with divided attention has been attributed to the back-and-forth shifting of an indivisible "spotlight" mechanism of spatial attention (Posner, 1980), and some neurophysiological studies of attention have found effects consistent with a spotlight switching between locations (Buschman and Miller, 2009; Landau and Fries, 2012). But Niebergall et al. (2011) failed to find evidence of attention switching between the two attended representations, suggesting instead that attention can be stably split between two loci.

A more direct approach to examining whether attention shifts between two stimuli is to record simultaneously from populations of neurons representing those stimuli. Cohen and Maunsell (2010) monitored attention-related modulations in both hemispheres during a spatial attention task and found no significant negative correlations between the two hemispheres, which should occur if attention switches between two visual field locations. However, their $80 \%$ valid cueing paradigm may have discouraged switching between stimuli (but see Busch and VanRullen, 2010; Fiebelkorn et al., 2013). Neutral cueing encourages attention to multiple stimuli and is therefore most likely to reveal neuronal signatures of switching attention.

We recorded simultaneously from populations of neurons in both hemispheres in visual area V4 in monkeys trained to detect an orientation change at one of two possible stimulus locations. We interleaved blocks of cued/uncued and neutrally cued trials within each recording session and measured changes in firing rate and population activity. We found that firing rates during the neutral condition were lower than those of the cued condition but higher than the uncued condition. Spike count correlations and the Fano factor in the neutral condition were similarly intermediate. Neuronal modulation across hemispheres was positively correlated in both the cued and neutral conditions. Variance in the neutral condition was indistinguishable from the cued condition and less than that expected if attention switched between stimulus locations. These results support the hypothesis that neuronal populations in area V4 can function in a distributed manner across hemispheres to represent cue validity during spatial attention.

\section{Materials and Methods}

Neurophysiological recording and analysis. Two adult male rhesus monkeys (Macaca mulatta) were each surgically prepared with a titanium headpost and a scleral eye coil for eye tracking. After months of training on the behavioral task, two multielectrode arrays of 48 electrodes each (Blackrock Microsystems; $6 \times 8$ array of $1 \mathrm{~mm}$ electrodes, $400 \mu \mathrm{m}$ pitch) were implanted into visual area V4 in both hemispheres. V4 was localized using stereotaxic coordinates and by locating the lunate and superior temporal sulci during surgery. Surgeries were performed under isoflurane anesthesia and accompanied by standard courses of analgesics (buprenorphine and flunixin), antibiotics (cefazolin), and anti- inflammatories (dexamethasone). The Institutional Animal Care and Use Committee of Harvard Medical School approved all animal procedures.

After array implantation and animal recovery, receptive fields of neurons were mapped using a procedure that presented Gabor stimuli spanning a range of orientations, spatial frequencies, sizes, and visual field locations. The azimuths and elevations of the receptive fields of the left and right microelectrode arrays were centered around $\left(2^{\circ},-7^{\circ}\right)$ and $\left(-7^{\circ},-4^{\circ}\right)$ for Monkey A, and $\left(6^{\circ},-7^{\circ}\right)$ and $\left(-6^{\circ},-3^{\circ}\right)$ for Monkey W. Electrode impendences typically were between 0.2 and $1 \mathrm{M} \Omega$ at $1 \mathrm{kHz}$.

Data collection was performed using a Blackrock Microsystems 128channel recording system. Single neurons were observed online for monitoring purposes. Single neurons and multiunit responses were isolated offline using spike-sorting software (Plexon). The best-isolated neuronal activity on each electrode (single-unit or multiunit) was used so that there was only one unit per channel from each day's recording. We recorded a total of 210 single-unit and 1598 multiunit responses (mean \pm SD per session: $8 \pm 3.2$ and $61 \pm 4.9$ ).

Behavioral task. For the primary task, monkeys were required to fixate throughout each trial on a small white fixation spot in the center of a screen $\left(\leq 1.8^{\circ}\right.$ square fixation window) and to respond when a Gabor with a different orientation appeared. Two counterphasing Gabors were always presented simultaneously and synchronously. At a random time, picked from an exponential distribution (e.g., minimum $500 \mathrm{~ms}$, mean $3000 \mathrm{~ms}$, maximum $5500 \mathrm{~ms}$ ) and constrained to fall on when the counterphasing Gabor was at $0 \%$ contrast (100 ms intervals), one Gabor changed its orientation slightly. The orientation-changed Gabor remained on the screen, and the animal was rewarded for making a saccade to the location of the changed stimulus between 100 and $550 \mathrm{~ms}$ after it appeared. Attention was cued to one stimulus location for a block of 50 trials using four instruction trials in which a small white spot $\left(0.45^{\circ}\right.$ radius) appeared for 50-100 ms where the stimulus change would occur (and was likely to occur in the upcoming block). During "neutral" blocks of trials, identical white spots were simultaneously presented at both stimulus locations during instruction trials. Within a cued block, the change occurred with $80 \%$ probability on the cued side and $20 \%$ probability on the uncued side. The stimulus change occurred with $50 \%$ probability at each stimulus location during the neutral blocks. Only one change occurred on each trial, and the animal was rewarded for detecting a change regardless of which side it occurred on. A total of 5\% of trials were catch trials in which no stimulus change occurred and the monkey was rewarded for maintaining fixation. Catch trials are excluded from all analyses reported here.

Odd-symmetric, full-contrast Gabor stimuli were sinusoidally counterphased at $10 \mathrm{~Hz}$. The size (Gabor $\sigma: 0.45^{\circ}-1.6^{\circ}$ ), spatial frequency $(0.3-2.5$ cycles/deg), and initial orientation of each Gabor were optimized for a selected neuron during each recording session. The stimuli were therefore not optimized for the majority of recording channels. Stimuli were displayed on a CRT monitor $(100 \mathrm{~Hz}$ frame rate, $1024 \times 768$ pixels, 32 bits/pixel, 8 bit DACs) on a uniform gray background. The monitor was calibrated to produce linear steps of luminance and positioned $57 \mathrm{~cm}$ from the subject. Eye position was sampled at $200 \mathrm{~Hz}$, and spike times were sampled at $1 \mathrm{kHz}$.

Neuronal data analysis. All measures of population activity were calculated using spike counts during the $200 \mathrm{~ms}$ before the orientation change. Firing rates were first averaged over trials for each electrode channel, and then population firing rates were calculated by averaging over the channels from both arrays in a particular condition. Spike count correlations were measured using the Pearson's correlation between the spike counts of simultaneously recorded pairs of neurons. Population measures of spike count correlations were computed across trials per neuron pair and then averaged over all pairs recorded in the same condition for both electrode arrays. The findings were unaffected by using the geometric mean (Kohn and Smith, 2005; Luo and Maunsell, 2015) rather than the arithmetic mean. Mean-matching of the Fano factor was performed using $20 \mathrm{~ms}$ windows stepped by $10 \mathrm{~ms}$ using the "variance toolbox" for MATLAB (Churchland et al., 2010). Bootstrap analyses used 1000 repetitions. The main effects were present in both hemispheres and in both animals, so data were combined. 
Attention axis. For each recording session, the average responses to correctly detected changes at the cued locations were connected by a line using linear discriminant analysis (the "attention axis") (Cohen and Maunsell, 2010; Mayo et al., 2015). Only trials when the changes occurred at the cued location and were correctly detected were used to define the axis, although neuronal responses from other trials (uncued and neutral trials, corrects and misses) were projected onto the axis in various analyses. The attention axis analysis was based on responses up to but excluding responses to the orientation change, so that stimuli were identical on every trial within a session. The axes from each session were normalized in analysis-specific ways to allow comparisons across sessions (see Results). To avoid biases (Mayo et al., 2015), the attention axes were constructed using responses occurring 0-200 ms before the orientation change and used to examine responses from different trial epochs (200-1000 ms before the orientation change). Responses from all conditions (cued, neutral, and uncued) were projected onto the attention axis. Findings reported here were unaffected by the type of averaging used to calculate variance (e.g., across-trials vs within-trials) of attention axis position, and the same trends were present, but weaker, when using only a single time point $200 \mathrm{~ms}$ before the stimulus change instead of $1 \mathrm{~s}$ of data.

\section{Results}

We manipulated the spatial attention of macaque monkeys and measured neuronal activity in area V4 during 26 recording sessions (Monkey A: 14 sessions; Monkey W: 12 sessions). We investigated how cue validity affects attention-related changes in neuronal activity and behavior. We used cued and uncued conditions (commonly referred to as "attended" and "unattended"), as well as a neutral condition in which the stimulus change was equally likely to occur at either location. Our goal was to test whether attention-related changes in neuronal activity were graded and more variable in the neutral condition relative to the cued and uncued conditions.

Monkeys were trained to detect a change in the orientation of either of two Gabor stimuli, located in opposite visual hemifields (Fig. 1A). While the monkeys fixated, two full-contrast, oddsymmetric Gabors appeared and sinusoidally counterphased at $10 \mathrm{~Hz}$. After 500-5500 ms (exponential function; mean $=3000$ $\mathrm{ms}$ ), one Gabor changed orientation. Monkeys were trained to quickly make a saccade to the stimulus that changed. The parameters of each Gabor were optimized for a selected unit before recording began and were fixed throughout each recording session.

Instruction trials were used before each block of $\sim 50$ trials and consisted of a white spot briefly flashed at one (cued and uncued blocks) or both (neutral blocks) stimulus locations before the Gabors appeared. When a single flashed cue appeared in instruction trials, it indicated with $100 \%$ reliability where the stimulus change would occur. After four correct instruction trials, a block of trials without flashed cues commenced. Instruction trials with a single cued stimulus location indicated that the orientation change would occur at that location on $80 \%$ of the trials in the subsequent block ("cued") and occur at the other stimulus location on $20 \%$ of the trials ("uncued"). Simultaneously cued stimulus locations indicated that the orientation change was equally likely to occur at either location ("neutral"). Blocks of trials were interleaved and balanced between stimulus locations.

We used six randomly interleaved orientation change magnitudes during each recording session to characterize the effects of attention on behavioral performance. Figure $1 B$ illustrates detection performance during a typical session. As expected, the monkey performed best in the cued condition (solid line) and worst in the uncued condition (dotted line). Performance in the neutral condition was intermediate (dashed line). Thus, performance in- creased as either cue validity increased or the size of the orientation change increased.

Our task design required that there were fewer stimulus changes at the uncued location $(20 \%)$ than at the cued location $(80 \%)$. Consequently, we sampled only two, relatively difficult orientation changes in the uncued trials to obtain a similar number of trials at each measured orientation change magnitude (Fig. $1 B$, gray band). The analyses that follow (see Figs. 1, 2, and 3, excluding Fig. 4) are limited to the second- and third-most difficult trials in each cueing condition to ensure that the results are attributable to cue validity and not differences in the size of orientation changes.

Differences were also observed in the animal's reaction times during the same day of recording (Fig. $1 C$ ). The monkey responded fastest when the target location had been cued $(316 \mathrm{~ms})$, less quickly in the neutral condition (323 ms), and slowest in the uncued condition ( $369 \mathrm{~ms}$; Kruskal-Wallis ANOVA test between the three groups, $p=0.012$ ). A greater difference between the neutral versus uncued conditions ("costs") than between the neutral versus cued conditions ("benefits"; resampling test, $p<$ 0.001 ) is consistent with results in humans using similar paradigms (Rizzolatti et al., 1987; Proverbio and Mangun, 1994; Mangun and Buck, 1998).

Across all recording sessions, we measured the percentage of correctly detected orientation changes and the corresponding reaction times for the second and third most difficult orientation changes for each cueing condition (Fig. $1 B$, gray band). As shown in Figure $1 D$, monkeys were best at detecting orientation changes when the location of the change was cued, intermediate when the cueing was neutral, and worst when the location was uncued (mean percentage correct for cued, neutral, uncued: $71.7 \%$, 62.7\%, and 45.8\%; Kruskal-Wallis ANOVA test, $p<0.001$; Monkey A: 66.6\%, 54.8\%, and 33.8\%, $p<0.001$; Monkey W: $77.5 \%, 72.0 \%$, and $59.8 \%, p=0.004)$. Reaction times were similarly graded across cueing conditions such that neutral reaction times were slower than cued reaction times by $7 \mathrm{~ms}$, but faster than uncued reaction times by $10 \mathrm{~ms}$ (Fig. $1 E$; mean/SD reaction time for cued, neutral, uncued: $311 / 74 \mathrm{~ms} ; 318 / 74 \mathrm{~ms}$; and 328/75 ms; Kruskal-Wallis ANOVA test, $p<0.001$; Monkey A: 311/64; 319/64 ms; and 336/64 ms; $p<0.001$; Monkey W: 311/83 ms; $317 / 80 \mathrm{~ms}$; and $322 / 81 \mathrm{~ms} ; p<0.001)$. Thus, neutral cueing yielded detection performance and reaction times intermediate to those of cued and uncued conditions, consistent with human experiments using similar cueing paradigms (Posner et al., 1980; Castiello and Umiltà, 1992; Mangun and Buck, 1998; Montagna et al., 2009).

During the same sessions, we recorded the neuronal activity of populations of V4 neurons in both hemispheres using extracellular multielectrode arrays. We analyzed the V4 activity that accompanied the graded behavioral performance across cueing conditions. In each trial, we sampled neuronal activity $200 \mathrm{~ms}$ before the orientation change to capture the animal's attentional state just before the unpredictable change but exclude responses to the change itself (Fig. 1A, gray shading) (Cohen and Maunsell, 2010). Because the orientation at each stimulus location was always the same before the change, all neuronal activity collected in a single session was in response to identical sensory stimulation, and we therefore attribute changes in neuronal activity to changes in spatial attention.

Figure 2 summarizes the average population activity in the three cueing conditions. Firing rates were highest in the cued condition, lowest in the uncued condition, and intermediate in the neutral condition (Fig. 2A; mean firing rates for cued, neutral, 
A
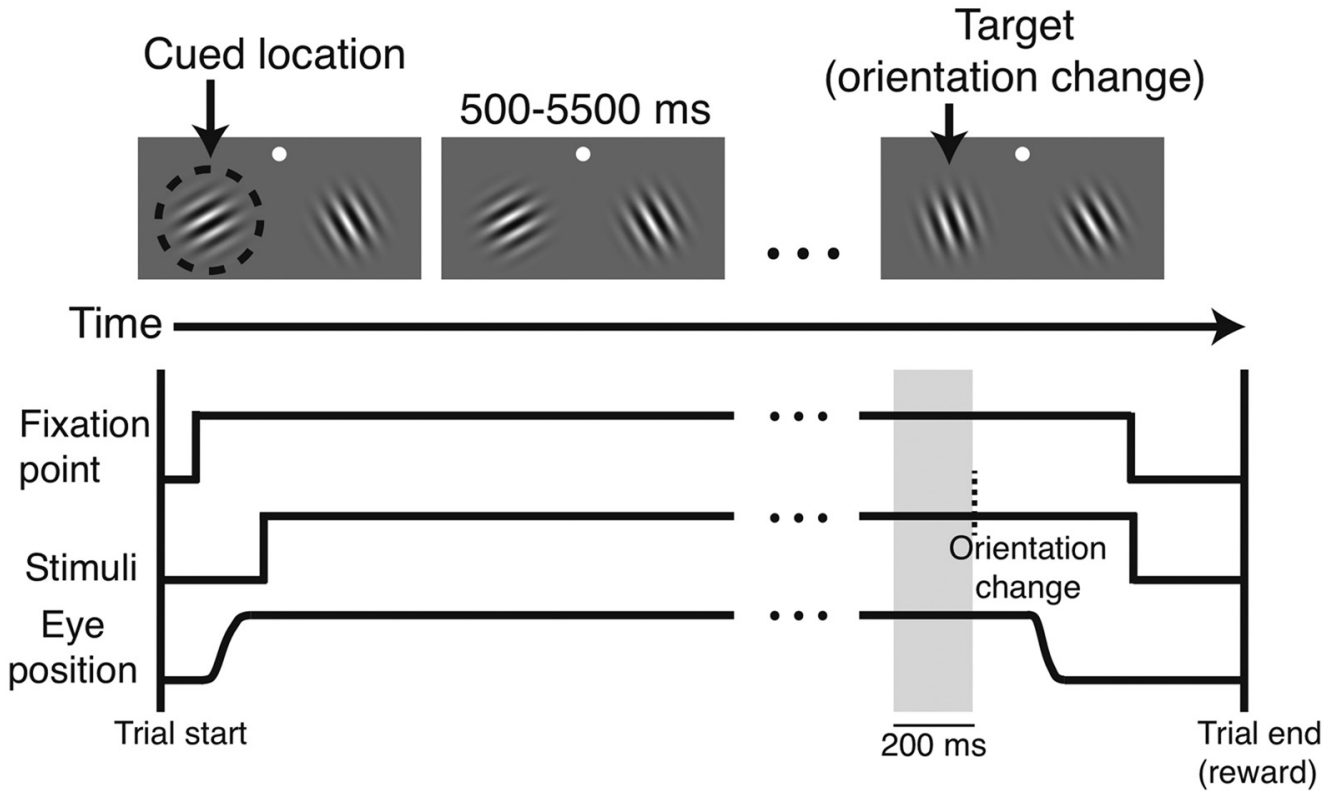

B

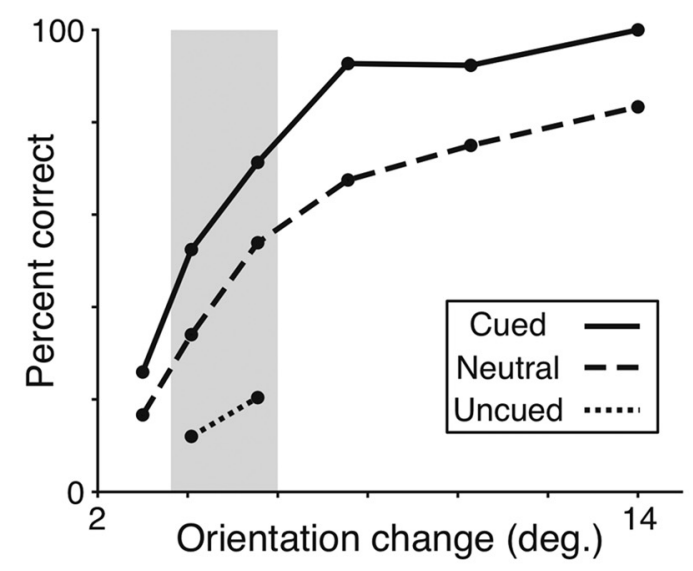

D

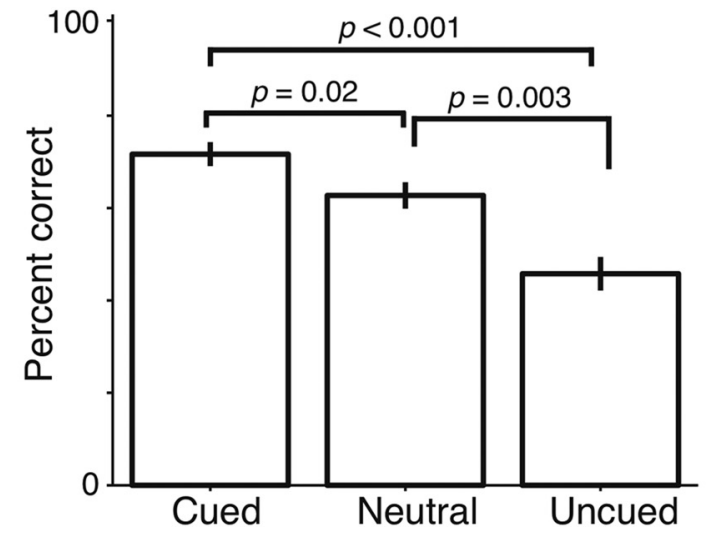

C
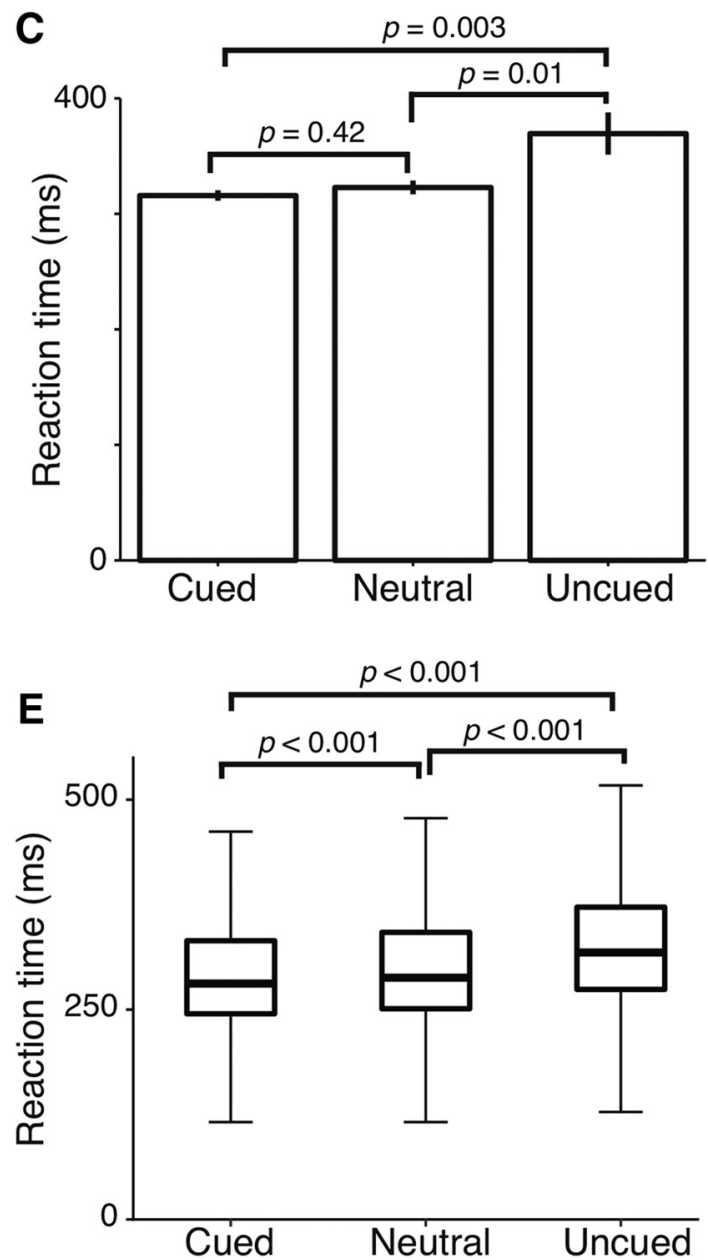

Figure 1. Detection performance and reaction times are graded across cueing conditions. A, Top, Visual display during task. White dot indicates the fixation point. Dashed circle (not visible during task) represents the location cued during instruction trials and, in this example trial, also the location of the orientation change. Bottom, Time course of task events. Vertical dashed line indicates the time of the orientation change. Gray band represents the $200 \mathrm{~ms}$ epoch just before the orientation change analyzed for Figures $1-3$ and $4 A$. B, Single-session detection performance as a function of the size of the orientation change for the three cueing conditions. Gray band represents the stimulus difficulty levels used for all subsequent analyses, except Figure 4 . C, Single-session reaction times for the stimulus levels in the gray band in $\boldsymbol{B}$. D. Mean detection performance across all 26 recording sessions. $\boldsymbol{E}$, Median reaction times (thick horizontal lines) and interquartile range (box) across all recording sessions; whiskers extend 1.5 times the interquartile range beyond either end of each box. $\boldsymbol{C}-\boldsymbol{E}$, Wilcoxon rank sum tests. $\boldsymbol{C}, \boldsymbol{D}$, Error bars indicate SEM. 

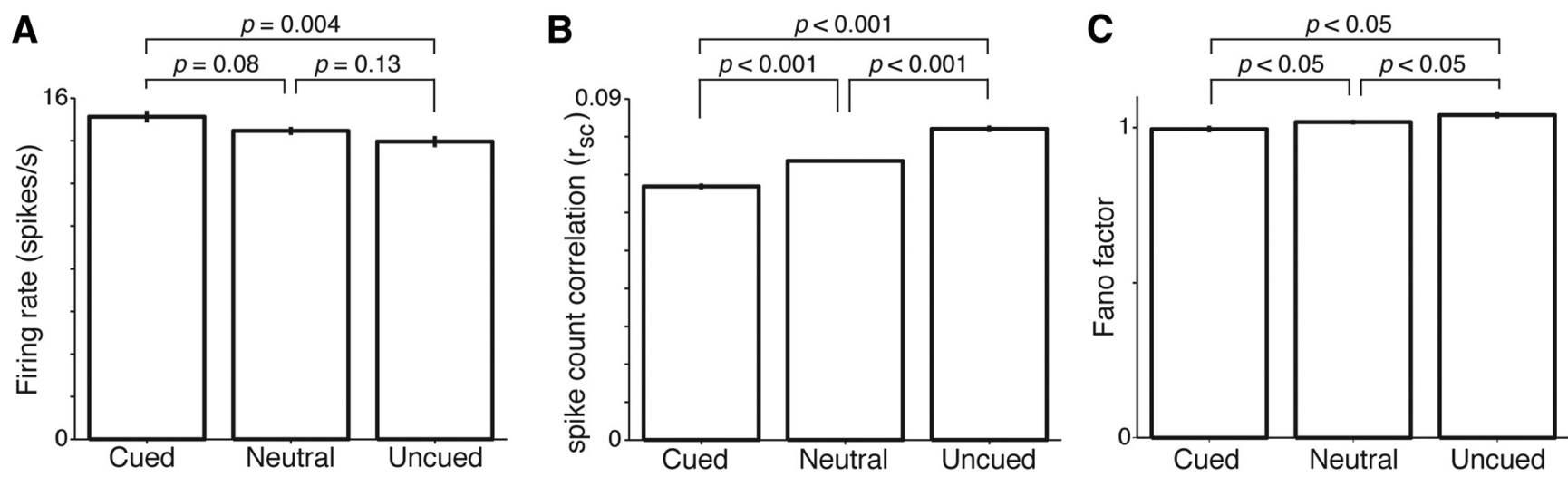

Figure 2. Population changes in neuronal activity related to attention are graded across cueing conditions. $\boldsymbol{A}$, Mean firing rates. $\boldsymbol{B}$, Mean spike count correlations. Wilcoxon rank sum tests performed in $\boldsymbol{A}, \boldsymbol{B}$. Error bars indicate SEM (not visible in some conditions). $\boldsymbol{C}$, Mean-matched Fano factor with $95 \%$ Cls. Time window for analyses was $200-0$ ms before the orientation change.

and uncued: 15.1, 14.5, and 14.0 spikes/s; Kruskal-Wallis ANOVA test, $p=0.02$; Monkey A: $17.8,16.7,16.0$ spikes/s, $p=$ 0.007; Monkey W: $12.4,12.1$, and 11.9 spikes/s, $p=0.48)$. Spike count correlations were correspondingly intermediate during neutral cueing, stronger than the correlations found in the cued condition and weaker than uncued correlations (Fig. 2B; mean spike count correlations for cued, neutral, and uncued: 0.067, 0.074, 0.082; Kruskal-Wallis ANOVA test, $p<0.001$; Monkey A: $0.101,0.117$, and $0.124, p<0.001$; Monkey W: $0.033,0.031$, and $0.041, p<0.001)$. Variance of the spike count correlations was largest in the uncued condition and similar in the cued and neutral conditions (SDs for cued, neutral, and uncued: 0.135, 0.133, $0.154 ; p \leq 0.01$ for all three pairwise comparisons using twosample $F$ test). Finally, the mean-matched Fano factor was lowest in the cued condition, intermediate in the neutral condition, and highest in the uncued condition. All conditions had nonoverlapping 95\% CIs (Fig. 2C; means for cued, neutral, and uncued Fano factors: $0.995,1.018,1.041$; Kruskal-Wallis ANOVA test, $p<$ $0.001)$. Attention-related neuronal modulations in the cued and uncued conditions (commonly referred to as "attended" and "unattended") are consistent with those previously reported for V4 (Maunsell and Cook, 2002; Cohen and Maunsell, 2009; Mitchell et al., 2009). Our neutral cueing data expand on previous results and demonstrate that attention-related changes in neuronal activity during neutral cueing are intermediate to "attended" and "unattended" levels.

To further characterize changes in neuronal activity, we evaluated population responses in a multidimensional space in which each dimension represents the neuronal activity associated with one recording electrode (mean of 70 channels per session). The two points corresponding to the average responses to cued, correctly detected changes at the two stimulus locations were connected by a line called the "attention axis" (Cohen and Maunsell, 2010; Mayo et al., 2015) (see Materials and Methods). Responses during trials from all conditions for a given recording session were projected onto the resulting line. This process yielded a single value representing the position on the attention axis for each trial, which can be used to estimate the monkey's state of attention (Cohen and Maunsell, 2010; Mayo et al., 2015).

To combine attention axis data across cueing conditions and days, the scaling of this axis was normalized so that the value for each trial was mapped onto an axis on which the two points corresponding to the average responses to cued, correctly detected changes at the two stimulus locations were assigned values of 1 and -1 . For each trial, a value of 1 was assigned to the side on which the change occurred, regardless of whether the change was cued, uncued, or neutral, or whether it was on the left or right side. A value of -1 was assigned to the side where no change occurred. Thus, cued trials, on which the animal had been instructed to attend to the side on which the change occurred, are expected to have values closer to 1 , and uncued trials, on which the animal detected a change to the side opposite to where it typically occurred, are expected to have values closer to -1 .

We first asked whether the average positions of trials on the axis associated with cued, neutral, and uncued trials were also graded, as was the case in the trial-averaged analyses of neuronal activity. Average attention axis positions differed systematically across the three cueing conditions (Fig. 3). As expected, neuronal responses on cued trials (leftmost bars), on which the animal had been validly cued to attend to the location where the change occurred, were closest to 1 . The values averaged $<1$ because they measure neuronal responses that were not used in constructing and normalizing the attention axis (Mayo et al., 2015). Correspondingly, neuronal responses on uncued trials (rightmost bars), on which the animal had been invalidly cued and was likely to be directing attention away from the location of the change, were closer to -1 . Neutrally cued responses were near 0 (center bars). Graded modulations in attention axis position were significant across conditions for both detected ("Hits") and missed stimulus changes (Kruskal-Wallis ANOVA test; both $p<0.001$ ). Thus, not only was the averaged population activity in the neutrally cued condition intermediate to the cued and uncued activity, but the location of neutrally cued trials in attentional state space, which combines activity from both hemispheres on each trial, was likewise intermediate.

In all three cueing conditions, neuronal responses were closer to 1 on detected changes ("Hits," white bars) than undetected changes ("Misses," gray bars). This confirms the utility of the attention axis because, regardless of mean location of attention, more hits are expected on those trials when attention happens to lie closer to the site where the change occurs ( 1 on the attention axis in Fig. 3).

Overall behavioral performance was correlated with attention axis position, in agreement with previous results using an independent dataset (Cohen and Maunsell, 2010; Mayo et al., 2015). Trials that fell on positions closer to 1 on the attention axis were associated with a higher detection performance than trials further from 1 ( $\chi^{2}$ test for cued, neutral, and uncued: Hit trials with projections greater vs less than 0 : all $p<0.001)$. Additionally, detect probability on the attention axis (analogous to choice 
probability) (Cohen and Maunsell, 2010) across conditions was $0.56 \pm$ 0.004 and significantly $>0.50$ (onesample $t$ test; $p<0.001$ ).

Intermediate neuronal responses during neutral cueing might arise if subjects gave sustained, partial attention to both sides throughout each trial, but might also arise from subjects alternating their full attention to each side, spontaneously switching sides either between trials or between intervals within a trial. Combined strategies (partial switching) might also occur. Although the idea of switching the focus of attention between locations, as opposed to maintaining divided attention across locations, has been a central issue in studies of visual spatial attention (Posner, 1980; Eriksen and St. James, 1986; Castiello and Umiltà, 1992), the evidence has been largely inferred from behavioral measures (Cave et al., 2010; Jans et al., 2010; but see McMains and Somers, 2004; Niebergall et al., 2011).

Multielectrode recordings in both hemispheres during the cued and neutral conditions allowed us to evaluate the possibility that the focus of attention switches between stimulus locations. Switching attention and divided attention make distinct predictions about fluctuations in attention between hemispheres. The switching hypothesis predicts that a unitary focus (a single spotlight) of attention moves between stimulus locations to detect the neutrally cued stimulus change (Posner et al., 1980; Eriksen and Yeh, 1985; Koch and Ullman, 1985; VanRullen, 2013). Switching of this sort requires that attention-related changes in neuronal activity are negatively correlated between cerebral hemispheres: when attention switches from one stimulus location to the other, neuronal responses will increase at one site and decrease at the other.

In the framework of our task, the switching hypothesis predicts inverse changes in attention allocated to the left and right stimuli (for detectable fluctuations in attention, $\sim<5 \mathrm{~Hz}$ ). Switching attention from one side to the other should cause positive changes in the activity of neurons in one hemisphere and negative changes in the activity of neurons in the other. Because the attention axis analysis combines the activity of many neurons, it provides a sensitive measure of the attention allocated toward a stimulus. When attention axes are constructed using only neurons from one cerebral hemisphere, they can provide independent, simultaneous measures of the attention allocated to each of two stimuli.

We constructed two separate attention axes using activity from each electrode array (Cohen and Maunsell, 2010; Mayo et al., 2015). To directly compare activity in the two hemispheres, for this analysis, the scaling of both axes was normalized so that the point corresponding to the average response to cued, correctly detected changes in the visual hemifield represented by the neurons being considered was assigned a value of 1 , and the point corresponding to the average response to cued, correctly detected changes in the other visual hemifield was assigned a value of -1 . With this assignment, shifting attention from one hemifield to the other should cause shifts of opposite sign on the attention axes for the two hemispheres. This negative correlation is imposed by the task structure and the way the axis is constructed when the cue alternates from one side to the other. The question we sought to answer was whether there were any acrosshemisphere correlations within cueing conditions.

Correlations in attention axis positions across hemispheres in the cued condition were small and slightly positive (Fig. $4 A$, white bar on left; one-sample $t$ test on resampled means, $p<0.001$ ). Earlier work using a similar approach found no significant correlations in V4 during valid cueing (Cohen and Maunsell, 2010), but this can be attributed to the greater statistical power provided by our experimental design (see Discussion). Coordinated switching of attention in the neutral cueing condition should lead to negative covariance that makes the correlation more negative in the neutral condition. We found no evidence for this. Instead, correlations in the neutral condition were significantly greater than zero (Fig. $4 A$, white bar on right; one-sample $t$ test on resampled means, $p<0.001)$ and not significantly different from across-hemisphere correlations in the cued condition (two-sample $t$ test on resampled means, $p=0.27$ ). The small, positive correlations in attention axis positions across hemispheres violated the expectations of a single, coordinated mechanism of attention spanning V4 in both hemispheres.

For comparison, we also built attention axes using half of the number of electrode channels per session but randomly sampled from within the same array (Cohen and Maunsell, 2010). These within-hemisphere correlations (Fig. 4A, black bars) were considerably larger in both conditions, presumably because neurons representing the same stimulus are subjected to the same topdown signals and have stronger interconnections.

We also investigated the more general hypothesis that variability in attention during neutrally cued trials is greater than in cued trials (Denfield et al., 2015). A unitary spotlight of attention that alternated between the cued-left and cued-right states (as 
A

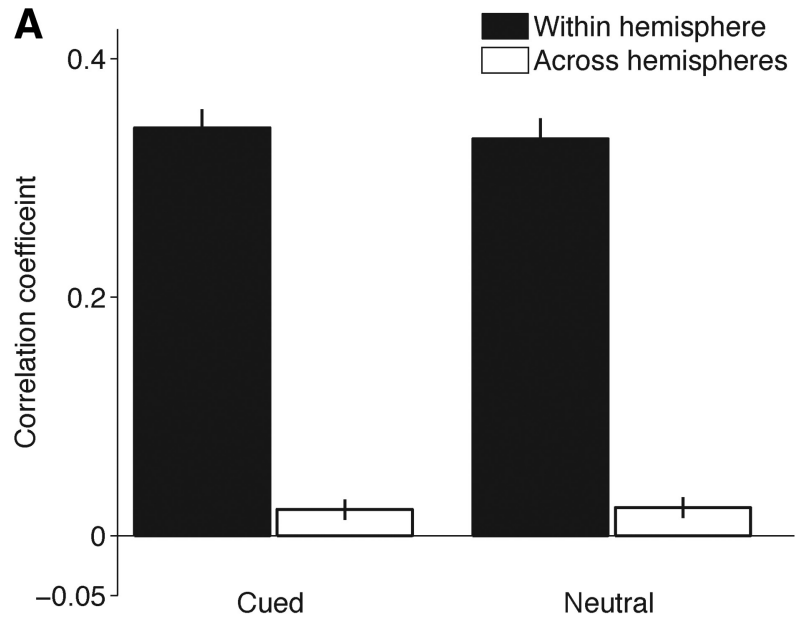

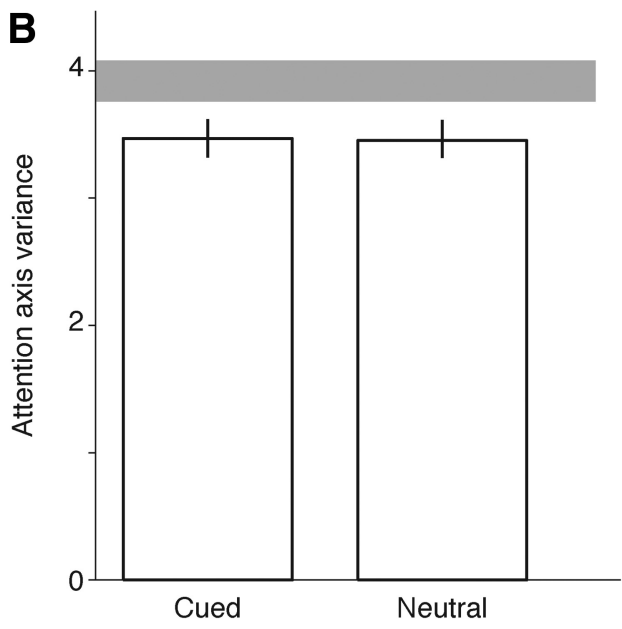

Figure 4. Covariance and variance in the cued and neutral conditions are indistinguishable. $A$, Mean correlations in attention axis positions for Hit responses from within the same hemisphere (black bars) and Hit responses from across hemispheres (white bars). $\boldsymbol{B}$, Mean variance in attention axis Hit positions in cued versus neutrally cued conditions. Gray band represents the range of variance expected in the Neutral condition by bootstrapping the Cued-left and Cued-right responses ( $95 \%$ Cls). Error bars indicate bootstrapped Cls. To improve statistical power, measures of variance included five 200 ms epochs before the period on which the attention axis was built (i.e., 1200 to 200 ms before the stimulus change), trials with all six orientation changes magnitudes, and were based on distributions trimmed to remove outliers (SD $=2.5,<7 \%$ of trials excluded).

measured by the attention axis built on all responses from both hemispheres) would produce more sample variance in the neutral condition than in a cued condition (Castiello and Umiltà, 1992; Niebergall et al., 2011). Even if no switching occurs, divided attention in neutral cueing might nevertheless be associated with less stable attention because of uncertainty regarding the target location (Pelli, 1985; Weichselgartner and Sperling, 1987). However, we found that variance in attention axis position was indistinguishable between the cued and neutral conditions (Fig. 4B; permutation test, $p=0.85)$. We estimated the variance that would be expected if attention had switched between the left and right stimuli by computing the variance of a combined cued-left and cued-right distribution. The gray band in Figure $4 B$ spans the 95\% CI for this variance, and it is substantially greater than the variance observed in the neutral condition. Thus, we found no evidence that variability in the state of attention was elevated during neutrally cued trials.

We also considered the fact that the phase of attentional fluctuations between stimulus locations may not be aligned with the phase of the stimulus cycle. In this case, we may not observe comparable levels of variance simply because of misaligned data. To address this issue, we repeated the same analysis and shifted the same duration window of neuronal activity at $10 \mathrm{~ms}$ intervals so that the attention axis was built on activity ending $200-400 \mathrm{~ms}$ before the stimulus change (two full stimulus cycles). This approach tests for other phase relationships between the allocation of attention and the stimulus cycle. The average variance in attention axis position during neutral cueing remained $<95 \%$ CIs obtained via bootstrapping the cued responses at various temporal alignments, consistent with Figure $4 B$. Thus, fluctuations in attention during neutral cueing were significantly less than expected if the monkey was switching attention between the two locations. Although it remains possible that attention might alternate between locations at frequencies that could not be detected with the $200 \mathrm{~ms}$ spike counting windows that we used, we found no evidence for switching frequencies up to $5 \mathrm{~Hz}$, consistent with previous results (Herrington and Assad, 2009; Horowitz et al., 2009; Niebergall et al., 2011).

\section{Discussion}

Neuronal modulations related to attention were graded across cued, neutral, and uncued conditions. This was observed in terms of averaged population measures, including rate of firing, Fano factor, and pairwise spike count correlations (Fig. 2), as well as when using a multidimensional approach to estimate the state of attention (Fig. 3). In all cases, neuronal activity during neutral trials was intermediate to that of cued and uncued trials, consistent with the idea that neuronal resources were distributed to both stimulus locations according to the demands of the task. More direct measures of the state of attention within and across hemispheres also failed to find evidence of a singular focus of attention. Cross-hemisphere correlations of attentional modulation were weak and slightly positive in both the cued and neutral conditions (Fig. 4A). Variability in spatial attention was also indistinguishable between the cued and neutral conditions (Fig. $4 B)$. These results suggest that the two hemispheres can function relatively independently during spatial attention, even during ambiguous cueing.

In contrast to the weak correlations in attention axis values that we found here, previous work by Cohen and Maunsell (2010) found no significant correlations between hemispheres using a similar task that included only cued and uncued conditions. Given the small correlations found here ( $\sim 0.02$; Fig. $4 A)$, the difference in results may stem from a variety of differences in task design, sample size, or behavioral strategies. We think that most of the difference can be attributed to greater statistical power in the current study. Our task design included a sustained stimulus, which provided many $200 \mathrm{~ms}$ epochs of spike counts per trial, as opposed to the single $200 \mathrm{~ms}$ epoch that was analyzed in the earlier study. The current analysis also involved more trials per session. Regardless of the small differences in reported values, the current results serve as a second independent dataset that fails to find the across-hemisphere correlations indicative of a unitary control of spatial attention.

Baruni et al. (2015) recently recorded from neurons in monkey V4 while manipulating attention by varying the relative size of rewards associated with stimuli in the left and right hemifields. 
Their design included different reward sizes on the two sides, similar to our cued and uncued conditions, and also trials with the same reward size on both sides, similar to our neutral cueing condition. As in our experiments, behavioral performance was best for stimuli on the side with a relatively large reward, worst on the side with a relatively small reward, and intermediate when the rewards were the same size on both sides. However, unlike the current results, their neutral condition did not produce intermediate responses from V4 neurons. Instead, when both sides had large rewards, V4 responses were indistinguishable from those to a stimulus associated with a large reward that had been paired with a small reward for the other stimulus. Correspondingly, when both sides had small rewards, V4 responses were indistinguishable from those to a stimulus associated with a small reward that had been paired with a large reward for the other stimulus. Baruni et al. (2015) suggested that absolute reward size, but not relative reward size, is a key determinant of neuronal modulation in V4.

Baruni et al. (2015) kept the probability of a reward being available fixed at $50 \%$ on each side, and modulated attention by adjusting reward sizes. The current study instead kept the absolute (and relative) reward sizes constant (and equal) on the two sides, and modulated attention by adjusting the probabilities that a reward would be available on each side. Thus, whereas absolute reward size might be a key determinant of neuronal modulation in V4, reward probability must be added to the list to key determinants. In the current study, V4 activity and behavioral performance covaried across the three cueing conditions. That is not the case for the data of Baruni et al. (2015), where behavioral performance was unchanged between large rewards on both sides and small rewards on both sides, but V4 activity varied with reward size. Identifying the full set of factors that affect V4 activity and behavioral performance in attention tasks will require additional, more elaborate, experiments.

Work from our laboratory recently demonstrated that the majority of neuronal modulation in V4 was associated with changes in behavioral sensitivity, but not changes in behavioral criteria (Luo and Maunsell, 2015). An analysis of the behavioral strategies of monkeys used in the current study revealed that they responded to cueing by adjusting both their sensitivity and criteria (data not shown). Presumably the attention-related modulations described were associated specifically with the changes in behavioral sensitivity, but a thorough characterization of the sources of these modulations would require a more sophisticated behavioral paradigm than the one used here.

Human psychophysical studies have used the neutral cueing condition for decades. In general, reaction times during neutral cueing are intermediate to cued and uncued reaction times (Posner, 1980; Castiello and Umiltà, 1992; Mangun and Buck, 1998; Giordano et al., 2009), and electrophysiological recordings in humans are also graded across cueing conditions (Mangun and Hillyard, 1990; Mangun and Buck, 1998). The implications of these results for the neuronal mechanisms of attention are difficult to interpret because of the spatially coarse nature of the measurements. Various objections regarding the number of stimuli and their durations (Mangun and Buck, 1998; Cave et al., 2010), experimental design (Montagna et al., 2009), and sufficient statistical power (McCormick et al., 1998) have been raised when evaluating psychophysical data.

We found clear changes in detection performance and reaction times across cueing conditions. Our dataset was strengthened by a number of factors. First, we collected thousands of trials per day during dozens of days of recording, and our subjects were well trained and highly motivated. Second, we used a speeded reaction time task with randomized trial lengths (500-5500 ms) and included catch trials, which helped minimize anticipation and false alarms. Finally, we cued attention in blocks of trials to maximize changes in behavioral performance associated with endogenous attention (Posner et al., 1980). These factors echo those suggested by Mangun and Buck (1998) to explain discrepancies between their results and smaller effects in their previous work (Proverbio and Mangun, 1994). Like the updated work of Mangun and Buck (1998), our results provide additional evidence in favor of graded behavioral performance across cueing conditions.

We measured changes in neuronal activity related to attention every $200 \mathrm{~ms}$, consistent with the timescale of attentional shifts indicated by previous work (Posner, 1980; Weichselgartner and Sperling, 1987; McMains and Somers, 2004; Carlson et al., 2006; Herrington and Assad, 2009; Horowitz et al., 2009; Cave et al., 2010). However, changes in the focus of attention may occur at rates faster than a few Hertz. Several studies have found periodic fluctuations in behavior or in broader measures of neural activity, such as local field potentials (VanRullen et al., 2007; Buschman and Miller, 2009; Wolfe et al., 2011; Landau and Fries, 2012; Baruni et al., 2015). We tested a conservative version of the sampling hypothesis that did not require periodic switching. We found equivalent variation in attention during validly cued and ambiguous neutral trials, and these levels did not reach those predicted by switching a single focus of attention between stimuli (Fig. 4) at frequencies below a few Hertz. Switching at higher frequencies would not have been detected in these experiments. It remains possible that switching will be found in other behavioral settings. More work is needed to address the issue of extremely rapid attentional switching, as well as attentional effects within, as opposed to across, visual hemifields. Each stimulus in our task was presented in a separate visual hemifield, which limits our ability to understand neutral cueing between retinotopically distinct locations within the same hemisphere as well as the so-called "zoom model" of attention (Eriksen and St. James, 1986; Niebergall et al., 2011). Evidence from human work suggests that attention can be divided within a hemisphere (McMains and Somers, 2004; Malinowski et al., 2007; Itthipuripat et al., 2013), consistent with our finding of intermediate neuronal modulations during neutral cueing.

Our results suggest that visual attention may be deployed piecemeal to multiple stimulus locations. When the stimulus change is likely to occur at a particular location, resources for that location are engaged, and the typical changes in neuronal activity attributed to attention are observed. In contrast, when the location of the stimulus change is unknown, attentional resources are allocated approximately equivalently between locations. This conclusion corroborates previous psychophysical demonstrations that spatial attention can be deployed according to task demands (Alvarez and Cavanagh, 2005; Giordano et al., 2009; Itthipuripat et al., 2013; Drew et al., 2014; Störmer et al., 2014) and may call into question the feasilibity of a winner-take-all mechanism for spatial attention (Koch and Ullman, 1985) and attentional priority maps (Maunsell, 2015). In all, these results suggest that spatial attention is not governed by a single, global neuronal mechanism, even in an ambiguous cueing condition where such a mechanism would most likely be detected. 


\section{References}

Alvarez GA, Cavanagh P (2005) Independent resources for attentional tracking in the left and right visual hemifields. Psychol Sci 16:637-643. CrossRef Medline

Baruni JK, Lau B, Salzman CD (2015) Reward expectation differentially modulates attentional behavior and activity in visual area V4. Nat Neurosci 18:1656-1663. CrossRef Medline

Busch NA, VanRullen R (2010) Spontaneous EEG oscillations reveal periodic sampling of visual attention. Proc Natl Acad Sci U S A 107:1604816053. CrossRef Medline

Buschman TJ, Miller EK (2009) Serial, covert shifts of attention during visual search are reflected by the frontal eye fields and correlated with population oscillations. Neuron 63:386-396. CrossRef Medline

Carlson TA, Hogendoorn H, Verstraten FAJ (2006) The speed of visual attention: What time is it? J Vis 6:1406-1411. CrossRef Medline

Carrasco M (2011) Visual attention: the past 25 years. Vision Res 51: 1484-1525. CrossRef Medline

Castiello U, Umiltà C (1992) Splitting focal attention. J Exp Psychol Hum Percept Perform 18:837-848. CrossRef Medline

Cave KR, Bush WS, Taylor TG (2010) Split attention as part of a flexible attentional system for complex scenes: comment on Jans, Peters, and De Weerd (2010). Psychol Rev 117:685-696. CrossRef Medline

Churchland MM, Yu BM, Cunningham JP, Sugrue LP, Cohen MR, Corrado GS, Newsome WT, Clark AM, Hosseini P, Scott BB, Bradley DC, Smith MA, Kohn A, Movshon JA, Armstrong KM, Moore T, Chang SW, Snyder LH, Lisberger SG, Priebe NJ, et al. (2010) Stimulus onset quenches neural variability: a widespread cortical phenomenon. Nat Neurosci 13: 369-378. CrossRef Medline

Cohen MR, Maunsell JH (2009) Attention improves performance primarily by reducing interneuronal correlations. Nat Neurosci 12:1594-1600. CrossRef Medline

Cohen MR, Maunsell JH (2010) A neuronal population measure of attention predicts behavioral performance on individual trials. J Neurosci 30: 15241-15253. CrossRef Medline

Connor CE, Gallant JL, Preddie DC, Van Essen DC (1996) Responses in area V4 depend on the spatial relationship between stimulus and attention. J Neurophysiol 75:1306-1308. Medline

Denfield G, Ecker A, Tolias A (2015) Correlated variability in population activity: noise or signature of internal computations? Neuroscience Meeting Planner, Washington, DC: Society for Neuroscience, 2015 Online: Program 372.305.

Drew T, Mance I, Horowitz TS, Wolfe JM, Vogel EK (2014) A soft handoff of attention between cerebral hemispheres. Curr Biol 24:1133-1137. CrossRef Medline

Eriksen CW, St. James JD (1986) Visual attention within and around the field of focal attention: a zoom lens model. Percept Psychophys 40: 225-240. CrossRef Medline

Eriksen CW, Yeh YY (1985) Allocation of attention in the visual field. J Exp Psychol Hum Percept Perform 11:583-597. CrossRef Medline

Fiebelkorn IC, Saalmann YB, Kastner S (2013) Rhythmic sampling within and between objects despite sustained attention at a cued location. Curr Biol 23:2553-2558. CrossRef Medline

Giordano AM, McElree B, Carrasco M (2009) On the automaticity and flexibility of covert attention: a speed-accuracy trade-off analysis. J Vis 9:30.1-30.10. CrossRef Medline

Herrington TM, Assad JA (2009) Neural activity in the middle temporal area and lateral intraparietal area during endogenously cued shifts of attention. J Neurosci 29:14160-14176. CrossRef Medline

Horowitz TS, Wolfe JM, Alvarez GA, Cohen MA, Kuzmova YI (2009) The speed of free will. Q J Exp Psychol (Hove) 62:2262-2288. CrossRef Medline

Itthipuripat S, Garcia JO, Serences JT (2013) Temporal dynamics of divided spatial attention. J Neurophysiol 109:2364-2373. CrossRef Medline

Jans B, Peters JC, De Weerd P (2010) Visual spatial attention to multiple locations at once: the jury is still out. Psychol Rev 117:637-684. CrossRef Medline

Koch C, Ullman S (1985) Shifts in selective visual attention: towards the underlying neural circuitry. Hum Neurobiol 4:219-227. Medline

Kohn A, Smith MA (2005) Stimulus dependence of neuronal correlation in primary visual cortex of the macaque. J Neurosci 25:3661-3673. CrossRef Medline
Landau AN, Fries P (2012) Attention samples stimuli rhythmically. Curr Biol 22:1000-1004. CrossRef Medline

Luo TZ, Maunsell JH (2015) Neuronal modulations in visual cortex are associated with only one of multiple components of attention. Neuron 86:1182-1188. CrossRef Medline

Malinowski P, Fuchs S, Müller MM (2007) Sustained division of spatial attention to multiple locations within one hemifield. Neurosci Lett 414 : 65-70. CrossRef Medline

Mangun GR, Hillyard SA (1990) Allocation of visual attention to spatial locations: tradeoff functions for event-related brain potentials and detection performance. Percept Psychophys 47:532-550. CrossRef Medline

Mangun GR, Buck LA (1998) Sustained visual-spatial attention produces costs and benefits in response time and evoked neural activity. Neuropsychologia 36:189-200. CrossRef Medline

Maunsell JH (2015) Neuronal mechanisms of visual attention. Annu Rev Vis Sci 1:373-391. CrossRef

Maunsell JH, Cook EP (2002) The role of attention in visual processing. Philos Trans R Soc Lond B Biol Sci 357:1063-1072. CrossRef Medline

Mayo JP, Cohen MR, Maunsell JH (2015) A refined neuronal population measure of visual attention. PLoS One 10:e0136570. CrossRef Medline

McAdams CJ, Maunsell JH (1999) Effects of attention on orientationtuning functions of single neurons in macaque cortical area V4. J Neurosci 19:431-441. Medline

McCormick PA, Klein RM, Johnston S (1998) Splitting versus sharing focal attention: comment on Castiello and Umilta (1992). J Exp Psychol Hum Percept Perform 24:350-357. CrossRef Medline

McMains SA, Somers DC (2004) Multiple spotlights of attentional selection in human visual cortex. Neuron 42:677-686. CrossRef Medline

Mitchell JF, Sundberg KA, Reynolds JH (2009) Spatial attention decorrelates intrinsic activity fluctuations in macaque area V4. Neuron 63: 879-888. CrossRef Medline

Montagna B, Pestilli F, Carrasco M (2009) Attention trades off spatial acuity. Vision Res 49:735-745. CrossRef Medline

Moran J, Desimone R (1985) Selective attention gates visual processing in the extrastriate cortex. Science 229:782-784. CrossRef Medline

Niebergall R, Khayat PS, Treue S, Martinez-Trujillo JC (2011) Multifocal attention filters targets from distracters within and beyond primate MT neurons' receptive field boundaries. Neuron 72:1067-1079. CrossRef Medline

Pelli DG (1985) Uncertainty explains many aspects of visual contrast detection and discrimination. J Opt Soc Am A 2:1508-1532. CrossRef Medline

Posner MI (1980) Orienting of attention. Q J Exp Psychol 32:3-25. CrossRef Medline

Posner MI, Nissen MJ, Ogden WC (1978) Attended and unattended processing modes: the role of set for spatial location. Modes Perceiving Processing Information 137:158.

Posner MI, Snyder CR, Davidson BJ (1980) Attention and the detection of signals. J Exp Psychol Gen 109:160. CrossRef

Proverbio AM, Mangun GR (1994) Electrophysiological and behavioral "costs" and "benefits" during sustained visual-spatial attention. Int J Neurosci 79:221-233. CrossRef Medline

Rizzolatti G, Riggio L, Dascola I, Umiltá C (1987) Reorienting attention across the horizontal and vertical meridians: evidence in favor of a premotor theory of attention. Neuropsychologia 25:31-40. CrossRef Medline

Somers DC, Dale AM, Seiffert AE, Tootell RB (1999) Functional MRI reveals spatially specific attentional modulation in human primary visual cortex. Proc Natl Acad Sci U S A 96:1663-1668. CrossRef Medline

Spitzer H, Desimone R, Moran J (1988) Increased attention enhances both behavioral and neuronal performance. Science 240:338-340. CrossRef Medline

Störmer VS, Alvarez GA, Cavanagh P (2014) Within-hemifield competition in early visual areas limits the ability to track multiple objects with attention. J Neurosci 34:11526-11533. CrossRef Medline

VanRullen R (2013) Visual attention: a rhythmic process? Curr Biol 23: R1110-R1112. CrossRef Medline

VanRullen R, Carlson T, Cavanagh P (2007) The blinking spotlight of attention. Proc Natl Acad Sci U S A 104:19204-19209. CrossRef Medline

Weichselgartner E, Sperling G (1987) Dynamics of automatic and controlled visual attention. Science 238:778-780. CrossRef Medline

Wolfe JM, Võ ML, Evans KK, Greene MR (2011) Visual search in scenes involves selective and nonselective pathways. Trends Cogn Sci 15:77-84. CrossRef Medline 